\title{
Biomolecular
}

Chemistry

Cite this: DOI: 10.1039/c0xx00000x

EMERGING AREA

www.rsc.org/obc

\section{Olefin Cross-Metathesis for the Synthesis of Heteroaromatic Compounds}

\author{
Timothy J. Donohoe, ${ }^{* a}$ John F. Bower ${ }^{a, b}$ and Louis K. M. Chan ${ }^{a}$ \\ Received (in $X X X, X X X)$ Xth $X X X X X X X X X 20 X X$, Accepted Xth $X X X X X X X X X 20 X X$ \\ ${ }_{5}$ DOI: 10.1039/b000000x
}

The olefin metathesis reaction has underpinned spectacular achievements in organic synthesis in recent years. Arguably, metathesis has now become the immediate choice for a carbon-carbon double bond connection. De novo routes to heteroaromatic compounds using the cross metathesis (CM) reaction have only recently emerged as an efficient strategy. This allows a convergent union of simple, but

10 functionalised, three- to four-carbon olefinic core building blocks, to generate furans, pyrroles and pyridines with a high degree of control over the substitution patterns in the product.

\section{Introduction}

Heteroaromatic compounds play a pivotal role in drug discovery. Consequently, the ability to synthesise these motifs in 15 a concise, efficient and regiocontrolled manner is of importance to medicinal chemistry and ultimately to the health and wellbeing of society (Figure 1a). ${ }^{1}$ Synthetic approaches to substituted heteroaromatic compounds rely upon either the modification of a

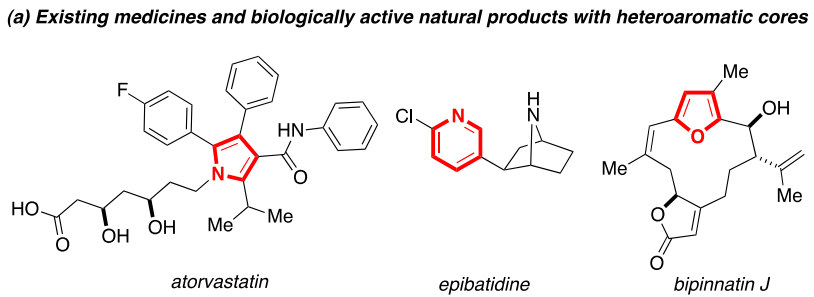

(b) Examples of modifications of existing heteroaromatic cores
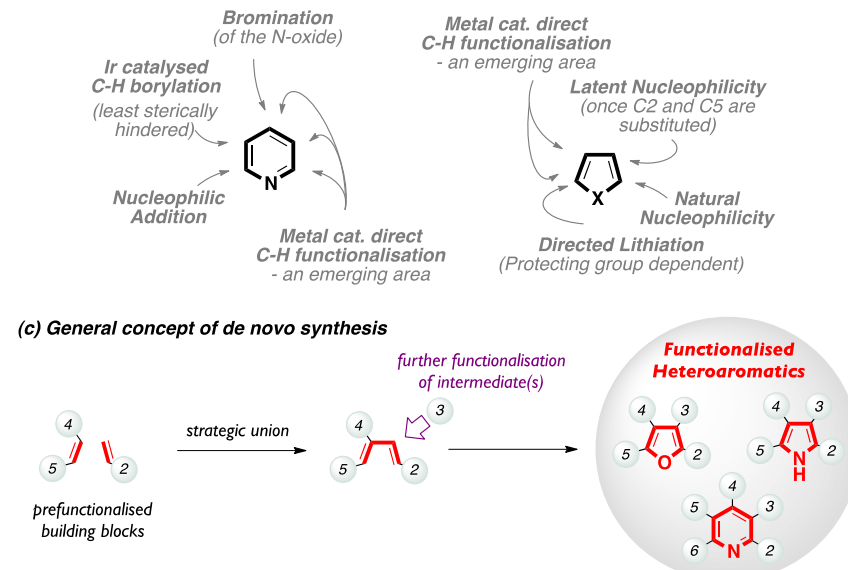

Figure 1. Heteroaromatics in chemistry.
20

pre-existing aromatic $\operatorname{core}^{2}$ (Figure $1 \mathrm{~b}$ ) or the implementation of de novo synthetic technologies (Figure 1c). ${ }^{3}$ Although these approaches are often employed in isolation, in the case of de novo syntheses, subsequent modification of the aromatic product is still ${ }_{25}$ possible. As such, a natural synergy emerges between the development of de novo methodologies and recent advances in metal catalysed cross-coupling. A major challenge in identifying strategies for de novo heteroaryl construction lies in providing efficient and predictable methods for regiodefined $\mathrm{C}-\mathrm{C}$ bond 30 formation. The ideal method should be operationally simple, rely upon minimal substrate prefunctionalisation and generate minimal quantities of waste.

${ }^{a}$ Department of Chemistry, University of Oxford, Chemistry Research 35 Laboratory, Mansfield Road, Oxford, OX1 3TA, U.K.; Tel: +44 (0) 1865 275649; E-mail: timothy.donohoe@chem.ox.ac.uk

${ }^{b}$ Current address: School of Chemistry, University of Bristol, Bristol, BS8 ITS, U.K.

40 Timothy J. Donohoe was an undergraduate in Chemistry at the University of Bath and then obtained his D.Phil. with S. G. Davies at the University of Oxford (1992). Following a 45 post-doctoral stay with P. D. Magnus in the USA, he joined the University of Manchester in 1994 as a lecturer and was promoted to Reader in 2000. In 2001, he joined the Dyson Perrins

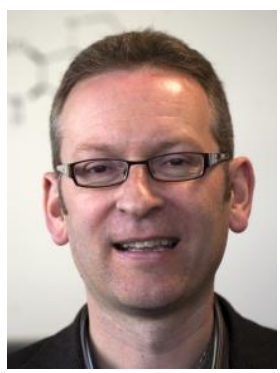

50 Laboratory, Oxford, as Lecturer in Chemistry and Fellow of Magdalen College. In 2004, he was appointed Professor of Chemistry at Oxford University. His research interests encompass asymmetric synthesis, the synthesis of aromatic compounds, total synthesis, catalysis and redox reactions. 


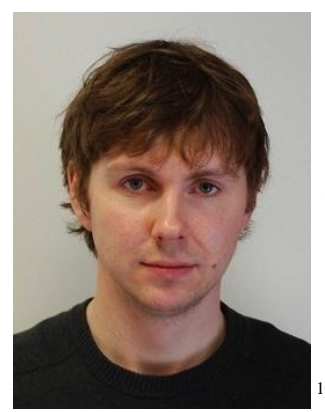

John F. Bower obtained his M.Sci. degree in Chemistry in 2003 from the University of Bristol. He then remained at Bristol to study for his 5 Ph.D. degree (2007) under the guidance of Professor Timothy $C$. Gallagher. His first postdoctoral appointment (2007-2008) was with Professor Michael Krische at the University of Texas at Austin. He then undertook a second postdoctoral appointment with Professor Timothy J. Donohoe at the University of Oxford, where he focussed on the use of olefin cross metathesis for heteroaryl synthesis. In 2010, he was awarded a Royal 15 Society University Research Fellowship and commenced his independent career at the University of Bristol.

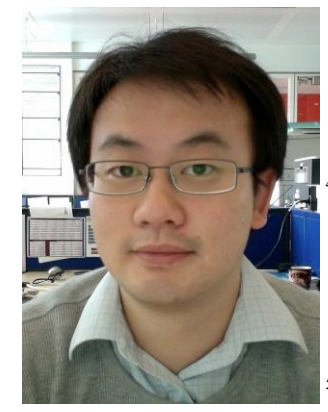

Louis K. M. Chan obtained his M.Sci. degree in Chemistry with a year in North America in 2006 from the University of Bristol, having carried out research with Professor James. P. Morken at the University of North Carolina at Chapel Hill, as well as with Professor Varinder K. Aggarwal at Bristol. He also obtained his Ph.D. degree (2010) under the supervision of Dr. Matthew J. Gaunt at the University of Cambridge. He is currently carrying out post-doctoral research work in the laboratory of Professor Timothy J. Donohoe at Oxford, in the area of olefin metathesis for heteroaryl 55 synthesis.

\section{Ring Closing Metathesis Based Strategies}

20 The olefin metathesis reaction is seemingly ideal as a key catalytic step for the construction of heteroaromatics, as it holds the criteria as a simple, yet powerful C-C double bond forming reaction. Commercially available and operationally robust catalysts such as Hoveyda-Grubbs $2^{\text {nd }}$ generation ${ }^{4}$ and Zhan $1 \mathrm{~B}^{5}$ 25 catalysts (Figure 2a) allow the union of two different olefins to provide a single more complex olefinic product.

(a) Commercially avaliable and robust metathesis catalysts relevant to these studies.

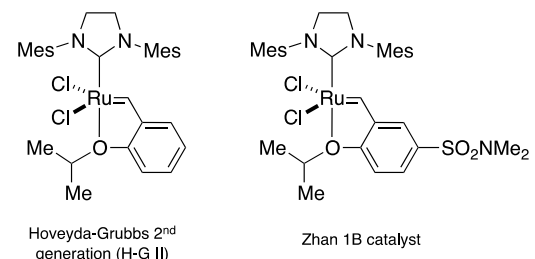

(b) A ring closing metathesis strategy for the synthesis of heteroaromatic compounds

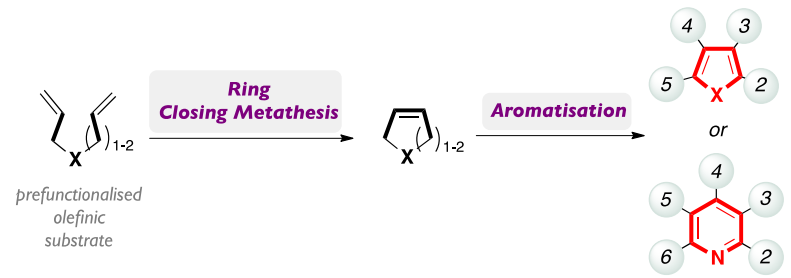

Figure 2. Ring closing metathesis for heteroaromatic synthesis.

30 Importantly, and of particular value to aryl synthesis, this method necessitates the provision of a product which possesses the unsaturation required for the eventual aryl target (Figure 2b). Despite this potential, heteroaryl methodologies that are reliant upon olefin metathesis have been slow to emerge. Although 35 early, isolated examples of metathesis based heteroaryl syntheses were reported, ${ }^{3 \mathrm{~h}, 3 \mathrm{i}, 6}$, systematic studies into the employment of this reaction for heteroaryl construction have only been undertaken more recently. Studies from our laboratory have focused upon developing intramolecular olefin metathesis 40 (termed Ring Closing Metathesis or RCM) based strategies (a) Pyridines via RCM:

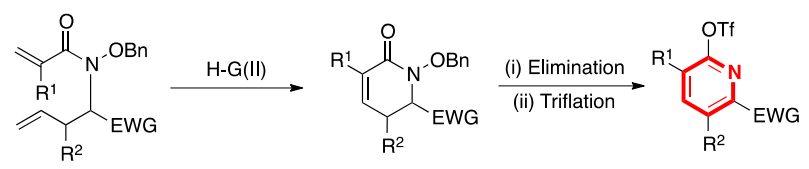

(b) Pyridazines via RCM:

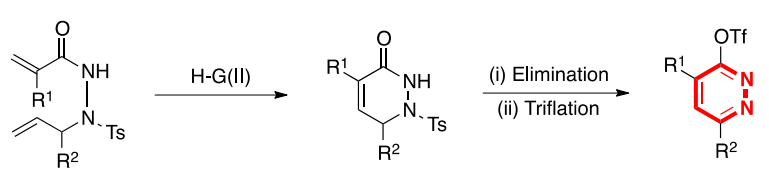

(c) Furans and Pyrroles via RCM:
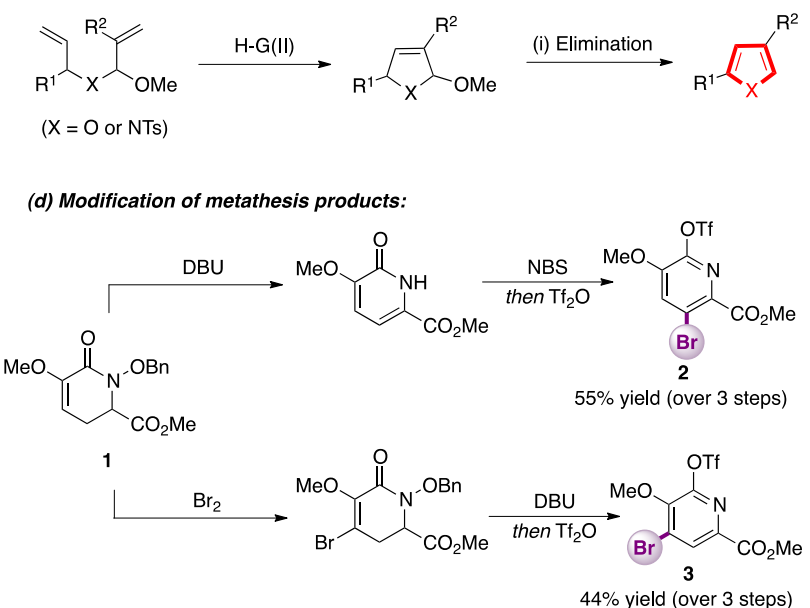

60 Scheme 1. Ring closing metathesis approaches to heteroaromatic compounds.

wherein the metathesis precursor embodies the oxidation level required for the eventual aromatic target. Accordingly, efficient 65 protocols for the synthesis of pyridines, pyridazines, pyrroles and furans have been developed in our $^{7}$ (Scheme 1a-c) and other ${ }^{8}$ laboratories.

In all of these cases, the key RCM event precedes aromatisation via elimination of a suitable leaving group under 70 either acidic or basic conditions. This chemistry has evident 
synthetic value as demonstrated by its application as a key step in the highly efficient synthesis of the furan cembranolide natural product (-)-deoxypukalide. ${ }^{9}$ A pertinent feature of these strategies is that further manipulation of the metathesis product, 5 either prior to or after aromatisation, enables access to higher heteroaryl substitution patterns. This is most aptly demonstrated by considering RCM product $\mathbf{1}$ which can be brominated either prior to or after elimination of the OBn group to provide either pyridine $\mathbf{2}$ or $\mathbf{3}$ in a regiodivergent manner (Scheme 1d).

10

\section{An Improved Approach Using Cross Metathesis}

In principal, the use of selective intermolecular olefin metathesis (termed Cross Metathesis or CM) represents an even 15 more powerful approach to heteroaryl construction. Here, there is no requirement for the design and preparation of a tethered metathesis precursor (Figure 3). Additionally, the key metathesis event is used both for the provision of the desired olefin and for the key fragment coupling step. As such, a scenario emerges 20 wherein $\mathrm{CM}$ is used to selectively couple two relatively simple olefinic starting materials with the goal of providing a single, more highly functionalised olefinic product which is then suitable for conversion to the target heteroaromatic.

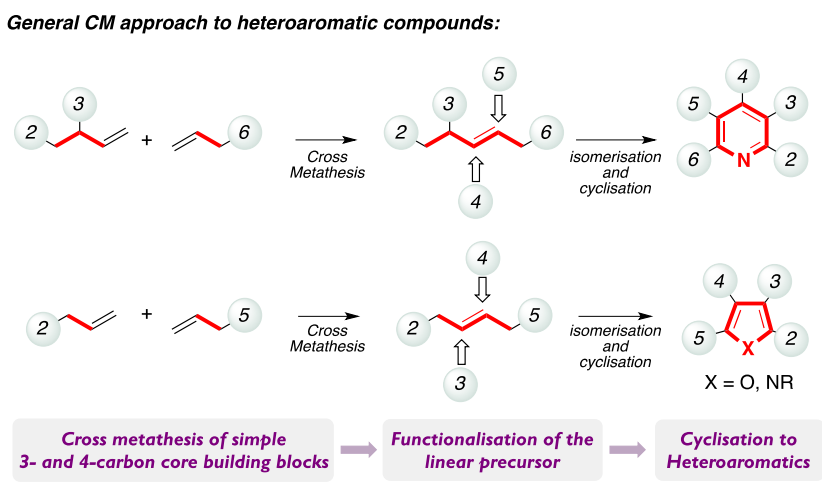

Figure 3. $\mathrm{CM}$ as an enabling approach to heteroaryl synthesis.

25

In order to implement CM for heteroaryl synthesis, two key points require addressing. The first involves identifying olefinic cross-coupling partners which can undergo selective crossmetathesis. As the use of CM in synthesis has gained pace, the 30 amount of empirical information available has reached levels where likely CM partners are readily identified. The likelihood of a successful $\mathrm{CM}$ can be predicted by considering which class an olefin belongs to as outlined in a key publication by Grubbs. ${ }^{10}$ The second point relates to identifying profitable strategies for 35 the conversion of a trans-olefin (metathesis product) to a cisolefin as required for the eventual aromatic target (vide infra).

\section{Cross-Metathesis Based Approaches to Furans}

40 Our early studies involved developing effective CM-based entries to substituted furans. ${ }^{11}$ Allylic alcohols have emerged as a special olefin class for metathesis, perhaps due to hydrogen bonding between the $-\mathrm{OH}$ and the chloride ligands associated with the ruthenium-based catalyst systems. ${ }^{12}$ Accordingly, these 45 species readily undergo $\mathrm{CM}$ with enones to provide $\gamma$ hydroxyenone intermediates $\mathbf{4}$ which are predisposed to cycloaromatise under acidic conditions. Using this chemistry as a basis, we developed a tandem protocol, that employs catalytic quantities of ruthenium and acid (PPTS) catalysts, to allow the 50 direct conversion of an allylic alcohol-enone pair to a furan (Scheme 2). This protocol is amenable to the employment of a diverse range of allylic alcohol or enone partners and delivers complex 2,5-disubstituted furans in moderate to excellent yield. In the examples shown in Scheme 2, olefin isomerisation is 55 promoted by an acid co-catalyst (PPTS).
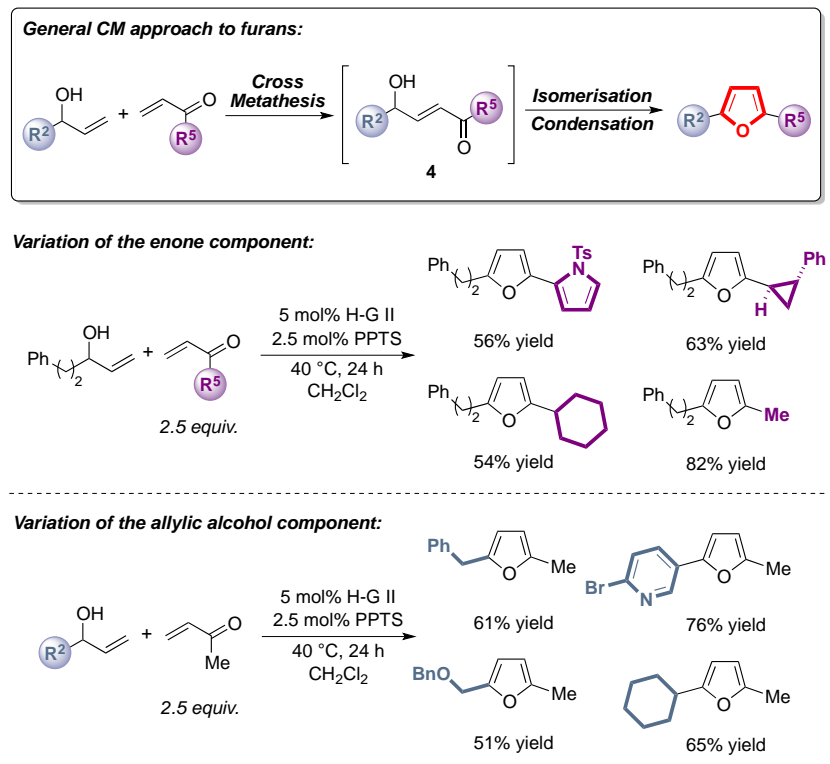

Scheme 2. CM approach to 2,5-disubstituted furans.

A more profitable strategy involves promoting isomerisation by using a protocol which facilitates concomitant introduction of ${ }_{60}$ further functionality. In this context, we have shown that Heck reaction of $\delta$-hydroxyenone intermediates 4 serves to introduce an aryl group onto the enone $\beta$-position and simultaneously effects olefin isomerisation to directly afford trisubstituted furans. Isomerisation of the enone from trans to cis under Heck 65 conditions is a result of the mechanistic requirements of the process (syn-carbopalladation followed by $s y n$ - $\beta$-hydride elimination), making the $\mathrm{CM} / \mathrm{Heck}$ combination an ideal one for our purpose. ${ }^{13}$ The opportunity to further functionalise the $\delta$ hydroxyenone metathesis products is of particular importance as ${ }_{70} \mathrm{CM}$ between 1,1-disubstituted allylic alcohol or enone partners is not efficient and so direct metathetic entries to trisubstituted furans are currently precluded. Using this CM-Heck tandem protocol, a variety of trisubstituted furans are available in short order and with complete levels of regiocontrol (Scheme 3a). A 75 powerful feature of this chemistry resides in the ability to dictate the final furan substitution pattern by programming the oxidation level of the allylic alcohol and enone CM precursors. Inversion of the oxidation level of this pair ultimately provides the alternative furan regioisomer (Scheme 3b).

80 Both of these approaches towards furans can be used to 
synthesise more complex polycyclic systems by rendering the processes intramolecular. Accordingly, tandem CMcycloaromatisation of tethered allylic alcohol-enone $\mathbf{5}$, under high dilution conditions, afforded macrocyclic furan $\mathbf{6}$, which is 5 structurally related to the core of the furancembranolide family of

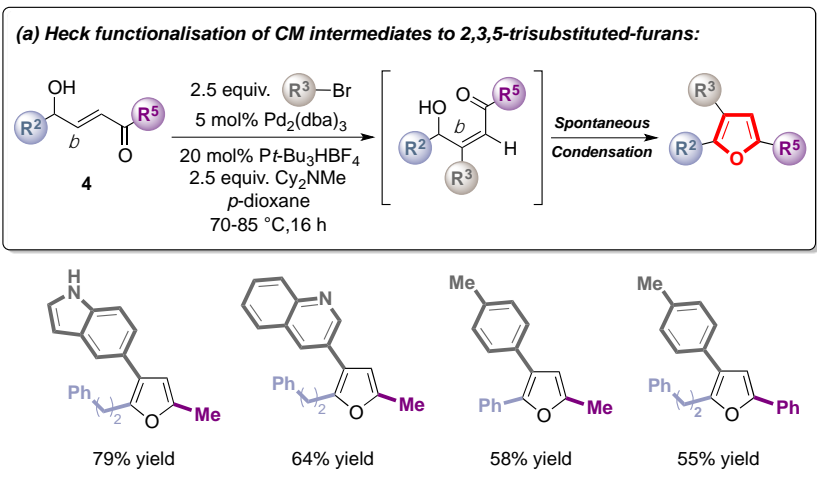

(b) Starting material oxidation level programmes regioselectivity:

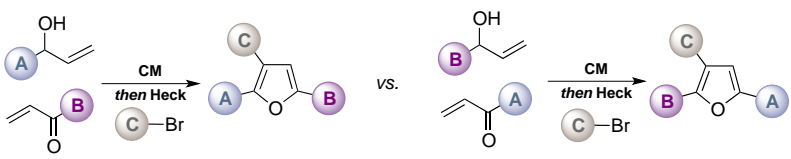

Scheme 3. CM-Heck approach to trisubstituted furans.

natural products, ${ }^{9}$ in excellent yield (Scheme 4a). This tandem 10 macrocylisation-aromatisation process unites the established utility of RCM for macrocycle synthesis with the heteroaryl methodology presented herein. Similarly, intramolecular Heck arylation of $\mathbf{7}$ affords furan $\mathbf{8}$ and potentially provides a blueprint for the construction of other complex, fused polycyclic ring 15 systems (Scheme $4 b$ ).

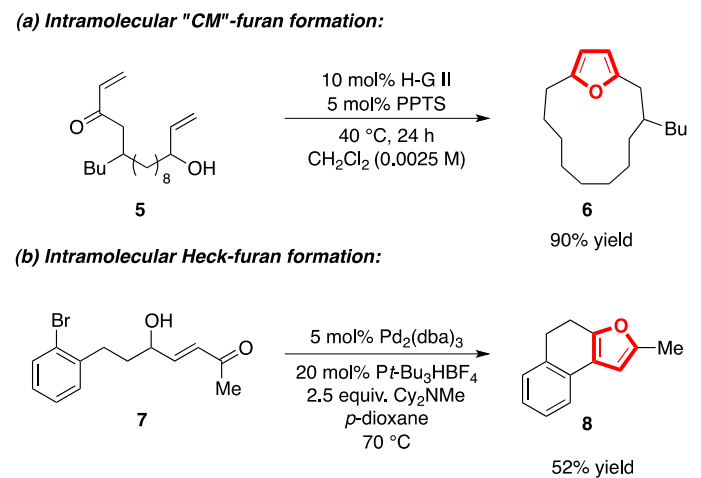

Scheme 4. Intramolecular "CM" or Heck reactions allow the formation of extra rings.

20

\section{Cross-Metathesis Based Approaches to Pyrroles}

Extension of the chemistry discussed so far to the synthesis of pyrroles represents a natural progression. Here, we anticipated ${ }_{25}$ that $\mathrm{CM}$ between suitably protected allylic amine and enone components would provide efficient access to $\gamma$-amino enones 9 which can then potentially be converted to pyrroles either upon treatment with acid or with a discrete Heck arylation step. ${ }^{14}$ An added complication of this scenario lies in the requirement to 30 protect the allylic amine component, which serves to prevent metathesis catalyst deactivation and also improves the synthetic accessibility and the utility of the final pyrrole. We observed that the CM of homoallylic amine derivatives with enones is more demanding than that involving the corresponding allylic alcohols. 35 Nevertheless, a variety of amino components underwent efficient $\mathrm{CM}$ with a range of enones to provide $\gamma$-amino enone intermediates 9 (Scheme 5). These were then converted to the final pyrrole upon exposure to $p$ - $\mathrm{TsOH}$ at $70{ }^{\circ} \mathrm{C}$.
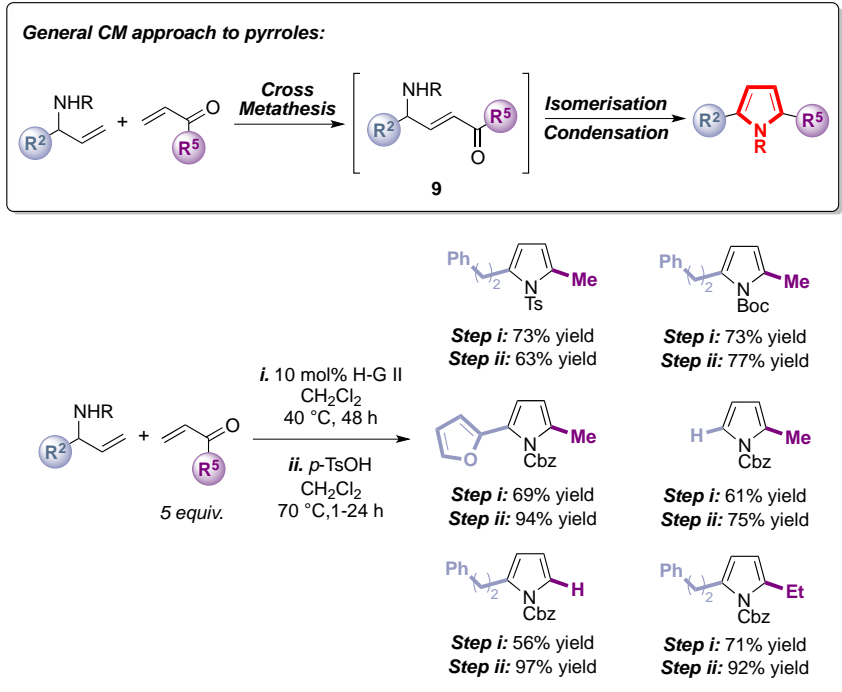

40 Scheme 5. CM approach to mono- and di-substituted pyrroles.

The more demanding nature of the $\mathrm{CM}$ process in these cases is evidenced by the requirement for (i) longer reaction times, (ii) higher catalyst loadings and (iii) our inability to develop an 45 efficient one pot CM-cycloaromatisation protocol. This latter point also eludes to the greater difficulty of cycloaromatisation of $\gamma$-aminoenones 9 compared to $\gamma$-hydroxyenones $\mathbf{4}$; in the examples presented in Scheme 5 a stronger acid ( $p$-TsOH vs. PPTS) and higher reaction temperatures $\left(70{ }^{\circ} \mathrm{C}\right.$ vs. $\left.40{ }^{\circ} \mathrm{C}\right)$ are 50 required.

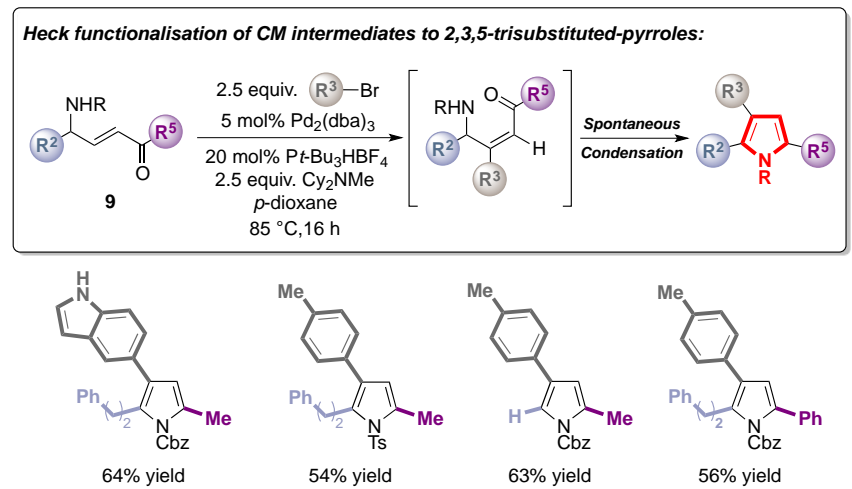

Scheme 6. CM-Heck approach to tri-substituted pyrroles.

Extension to the corresponding trisubstituted pyrroles using a 
tandem Heck-aromatisation process is also possible. Accordingly, diverse and highly complex trisubstituted pyrroles are available in short order and with complete levels of regiocontrol (Scheme 6). Note that this process also is tolerant of a range of protecting 5 groups at nitrogen (e.g. Cbz, Boc, Ts). Grela and co-workers have further demonstrated a one-pot synthesis of substituted pyrroles using CM. ${ }^{15}$ This was made possible with the use of $\mathrm{B}(\mathrm{OPh})_{3}$ as Lewis Acid for promoting isomerisation of the olefin geometry, as well as the subsequent cyclisation.

\section{${ }_{10}$ Cross-Metathesis Based Approaches to Pyridines}

As previously discussed, a powerful aspect of CM-based de novo heteroaryl synthesis is the ability to control the final substitution patterns during fabrication of the olefin precursors. In 15 the case of pyridines, appropriate choice of the starting CM partners, reaction conditions and reaction sequence allows the selective synthesis of mono-, di-, 2,3,6-tri-, 2,4,6-tri-, and 2,3,5,6tetra-substituted pyridines. ${ }^{16}$

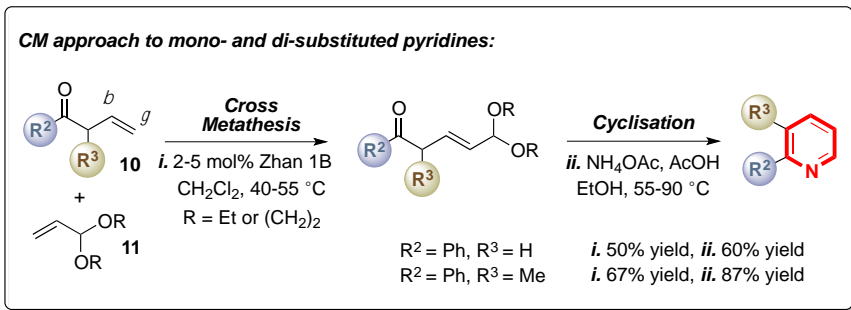

Scheme 7. CM approach to mono- and di-substituted pyridines.

20

The preparation of mono- and di-substituted pyridines, without substitution at $\mathrm{C} 6$, is readily achieved. In these cases, CM between $\beta, \gamma$-unsaturated ketones $\mathbf{1 0}$ and acetal protected acrolein derivatives $\mathbf{1 1}$ was most efficient (Scheme 7). Conversion 25 directly to the pyridine then occurs upon treatment with ammonia under mildly acidic conditions (which also effects acetal hydrolysis). Protection of the acrolein partner is important to circumvent stability issues associated with aldehydic unsaturated 1,5-dicarbonyls.
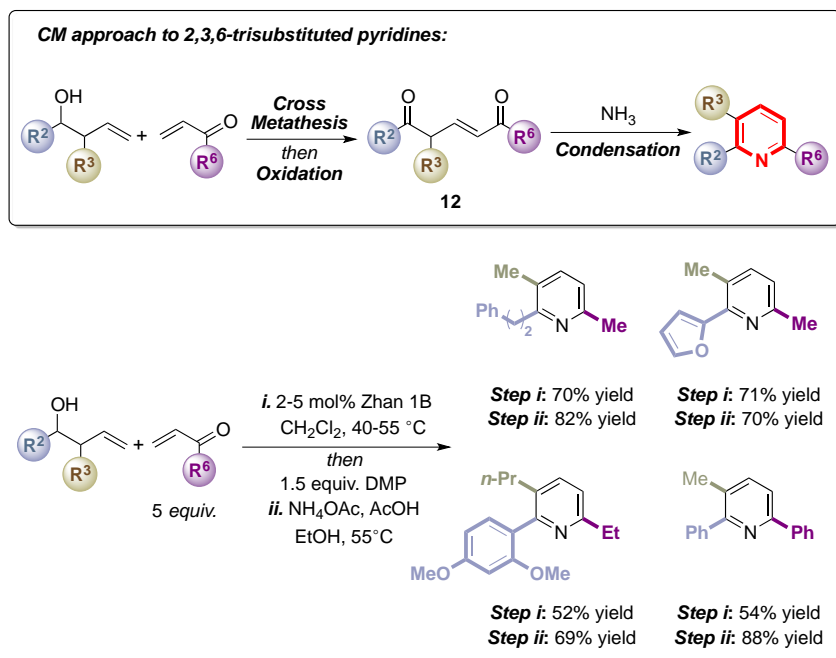

30

Scheme 8. CM approach to 2,3,6-trisubstituted pyridines.
Unsaturated 1,5-diketones are already established as efficient precursors to pyridines but have been underutilised for this purpose. We have shown that cross metathesis between 35 homoallylic alcohols and vinylketones follow by DMP oxididation is possible (Scheme 8). The trisubstituted unsaturated 1,5-dicarbonyls 12 which were then converted to the final 2,3,6trisubstituted pyridines targets by exposure to ammonia. Note that during condensation, isomerisation of the double bond 40 established during the $\mathrm{CM}$ event is facilitated by the acidity of the dicarbonyl intermediates.

Investigation into other pyridine formation led to a route for the regiodefined synthesis of 2,4,6-trisubstituted pyridines. CM between a homoallylic sulfonamide and a vinylketone partner 45 provided access to $\delta$-amino enone intermediates $\mathbf{1 3}$ (Scheme 9). Upon Heck arylation, olefin geometry switching occurrs and this facilitates in situ acid promoted condensation to 14. Subsequent base induced aromatisation (by elimination of the sulfinate group) then provides the target pyridines. ${ }^{17}$ This latter process is 50 substrate dependant and the final elimination is either conducted in situ (with DBU) or in a separate step (with KHMDS or $\mathrm{KOH} / \mathrm{EtOH})$.
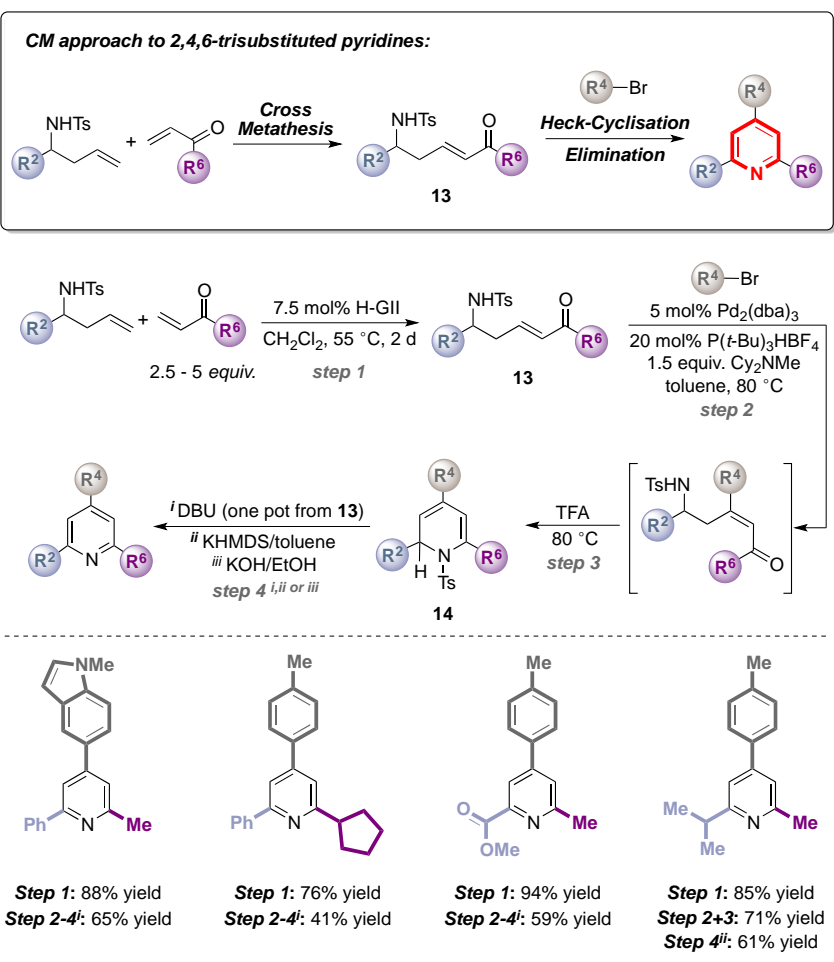

Scheme 9. CM-Heck approach to 2,4,6-trisubstituted pyridines. 55

Finally, access to higher pyridine substitution patterns is achievable via modification of the key 1,5-dicarbonyl intermediates described earlier. Accordingly, tetrasubstituted 60 pyridines are available via $\mathrm{Pd}$-catalysed $\alpha$-arylation (Scheme 10a) or base promoted $\alpha$-alkylation (Scheme 10b) of the key 1,5dicarbonyl 12. In both cases, complete levels of regiocontrol are observed and complex pyridine derivatives are available in a very short and modular manner. 


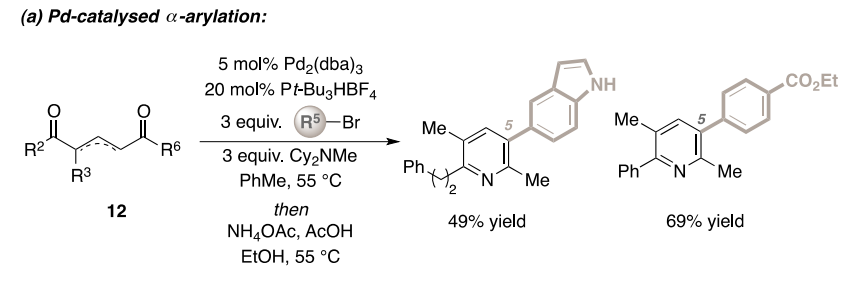

(b) Base promoted $\alpha$-alkylation:

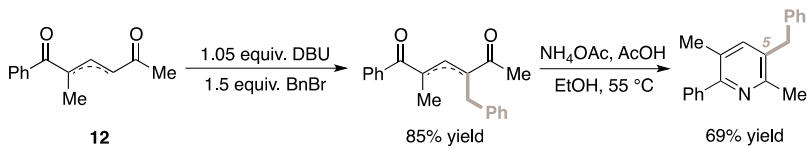

Scheme 10. Higher substituted pyridines via modification of the unsaturated 1,5-dicarbonyl intermediates.

\section{${ }_{5}$ Applications in Synthesis}

The validation of new methodologies in target directed settings is essential in demonstrating the true value and flexibility of the process. We have chosen to exemplify these new CM-based 10 heteroaromatic methodologies by applying them to two distinct targets, one medicinal and one natural product.

The first target is the core of the world's largest selling drug Atorvastatin 15 (or Lipitor) (Scheme 11). ${ }^{18}$ The key aromatic portion of this molecule possesses a fully substituted pyrrole core 15 and provides the ideal test rig for CM-based pyrrole forming methodologies. Accordingly CM of allylic amine derivative 16, which is available in two steps, with enone 17, afforded aminoenone 18. $\gamma$-Arylated enones of this type have proven to be challenging substrates for Heck-aromatisation. Nevertheless, after 20 optimisation trisubstituted pyrrole 19 was procured in $56 \%$ yield. $\mathrm{Cbz}$ deprotection then enabled further functionalisation of the remaining pyrrole $\mathrm{C}-\mathrm{H}$ by Friedel-Crafts acylation with phenyl isocyanate. Thus, the fully functionalised pyrrole core of Atorvastatin $\mathbf{1 5}$ is available with complete regiocontrol and in 6 25 steps from commercial materials. While this approach is, of course, not comparable to commercial routes to $\mathbf{1 5}$, one should recognize that highly flexible, modular and predictable entries to diverse pyrroles, and indeed many heteroaromatics, are likely to be of high utility in medicinal chemistry.
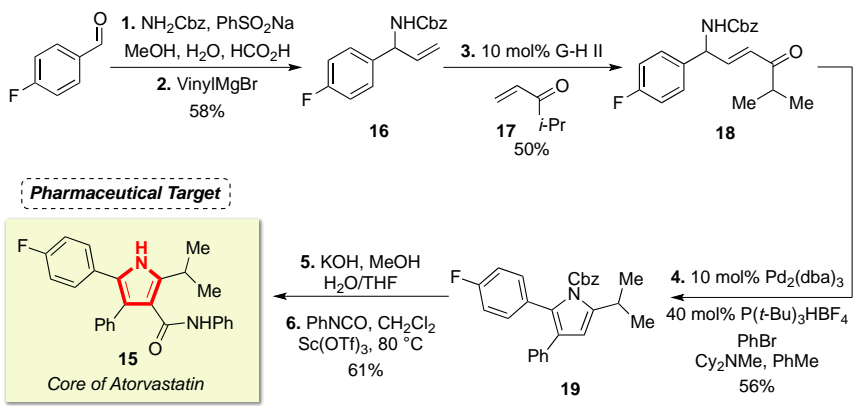

Scheme 11. CM-Heck approach to the pyrrole core of Atorvastatin.
The second target we have studied is the macrocycle $(R)-(+)-$ 35 muscopyridine which possesses an pyridine core embedded in a 13-membered macrocycle (Scheme 12). Here, we have employed an intramolecular variant of our CM-condensation pyridine methodology which facilitates the concomitant formation of the key macrocycle and pyridine portions. Given the utility of olefin 40 metathesis for macrocycle formation it is unsurprising that this reaction has been employed previously for syntheses of $\mathbf{2 0}$. However, in all previous approaches ${ }^{19}$ the element of unsaturation established during RCM (i.e. the olefin) was later reduced and not incorporated into the more natural site of the 45 pyridine. Commercially available undecenal 21 was subjected to Wadsworth-Emmons olefination and then asymmetric copper catalysed conjugate addition of $\mathrm{MeMgBr},{ }^{20}$ which gave excellent levels of enantioenrichment ( $>95 \%$ e.e.), before advancing to the key metathesis precursor 23. Metathetic macrocyclisation under 50 high dilution conditions and treatment of the crude product with ammonia then afforded directly $(R)-(+)$-muscopyridine 20 in $42 \%$ yield. The overall route to $\mathbf{2 0}$ comprises 8 steps and proceeds in $17 \%$ overall yield.
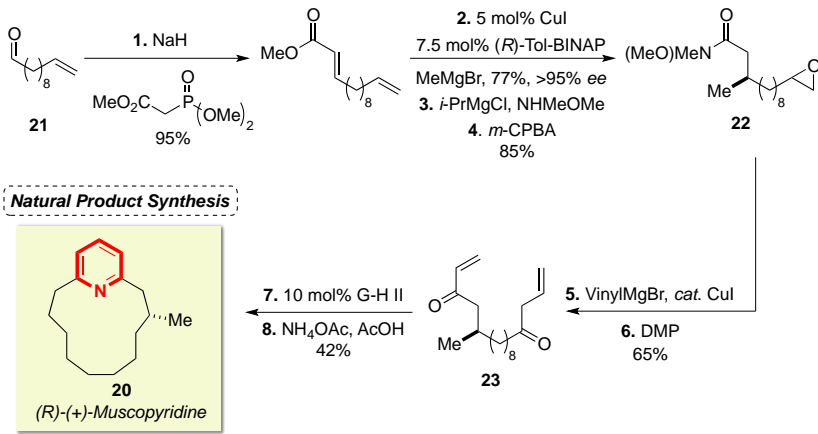

55

Scheme 12. Intramolecular "CM"-condensation approach to $(R)$ muscopyridine.

\section{Conclusions and Outlook}

60

Heteroaromatic compounds continue to provide a major synthetic challenge to the organic chemist and this has inspired a variety of creative methods for their regiocontrolled preparation. Stimulated by the synthetic power of the olefin cross-metathesis 65 reaction, we have delineated versatile strategies for the synthesis of a range of heteroaromatics, namely furans, pyrroles and pyridines. In all of these cases, the $\mathrm{CM}$ reaction provides the key by allowing the rapid and regiocontrolled union of simple precursors to provide a single more complex product. This CM 70 product may then be advanced directly to the aromatic target or further modified to provide access to more highly substituted derivatives. It is clear that as the power of the $\mathrm{CM}$ reaction increases then so will the number of potential CM-based avenues to heteroaromatic derivatives. Studies towards this broad goal 75 will be a focus of continuing efforts within our laboratory. 


\section{Notes and references}

1 (a) Pharmaceutical Chemistry. Drug Synthesis, Vol. 1.; H. J. Roth and A. Kleemann Eds.; Prentice Hall Europe: London, 1988; p 407. (b) V. Bonnet, F. Mongin, F. Trécourt, G. Breton, F. Marsais, P. Knochel and G. Queguiner, Synlett, 2002, 1008.

2 For reviews on functionalisation of heteroaryls: (a) J. C. Lewis, R. G. Bergman and J. A. Ellman, Acc. Chem. Res., 2008, 41, 1013. (b) I. J. S. Fairlamb, Chem. Soc. Rev., 2007, 36, 1036.

3 Reviews on furan synthesis: (a) S. F. Kirsch, Org. Biomol. Chem., 2006, 4, 2076. (b) X. L. Hou, H. Y. Cheung, T. Y. Hon, P. L. Kwan, T. H. Lo, S. Y. Tong and H. N. C. Wong, Tetrahedron, 1998, 54, 1955. For recent examples of furan synthesis: (c) C. K. Jung, J. C. Wang and M. J. Krische, J. Am. Chem. Soc., 2004, 126, 4118. (d) A. S. Dudnik and V. Gevorgyan, Angew. Chem. Int. Ed., 2007, 46, 5195. (e) J. Barluenga, L. Riesgo, R. Vicente, L. A. López and M. Tomás, J. Am. Chem. Soc., 2008, 130, 13528. (f) Y. Xiao and J. Zhang, Angew. Chem. Int. Ed., 2008, 47, 1903. (g) M. Zhang, H. F. Jiang, H. Neumann, M. Beller and P. H. Dixneuf, Angew. Chem. Int. Ed., 2009, 48, 1681. For recent reviews on pyridine synthesis: (h) G. D. Henry, Tetrahedron, 2004, 60, 6043. (i) M. Hill, Chem. Eur. J., 2010, 16, 12052. For recent pyridine synthesis: (j) O. G. Dediu, N. A. M. Yehia, T. Oeser, K. Polborn and T. J. J. Müller, Eur. J. Org. Chem. 2005, 1834. For selected recent examples of pyrrole synthesis: (k) R. Martin, C. H. Larsen, A. Cucenca, S. L. Buchwald, Org. Lett., 2007, 9, 3379. (1) S. Shafi, M. Kędziorek, K. Grela, Synlett, 2011, 124. (m) L. Ackermann, R. Sandmann, L. T. Kasper, Org. Lett., 2009, 11, 2031. (n) M. Yoshia, M. Al-Amin, K. Shishido, Synthesis, 2009, 2454. (o) P. W. Davies, N. Martin, Org. Lett., 2009, 11, 2293.

4 S. B. Garber, J. S. Kingsbury, B. L. Gray and A. H. Hoveyda, J. Am. Chem. Soc., 2000, 122, 8168.

5 Z.-Y. J. Zhan, U.S. Patent, 2007, 20070043180.

6 For a comprehensive review: W. A. L. van Otterlo, C. B. de Koning, Chem. Rev., 2009, 109, 3743 and references therein.

7 (a) T. J. Donohoe, J. F. Bower, J. A. Basutto, L. P. Fishlock, P. A. Procopiou and C. K. A. Callens, Tetrahedron, 2009, 65, 8969. (b) T. J. Donohoe, L. P. Fishlock, J. A. Basutto, J. F. Bower, P. A. Procopiou and A. L. Thompson, Chem. Commun., 2009, 45, 3008. (d) T. J. Donohoe, N. M. Kershaw, A. J. Orr, K. M. P. Wheelhouse (nee Gosby), L. P. Fishlock, A. R. Lacy, M. Bingham and P. A. Procopiou, Tetrahedron, 2008, 64, 809. (e) T. J. Donohoe, L. P. Fishlock and P. A. Procopiou, Org. Lett., 2008, 10, 285. (g) Donohoe, T. J.; Orr, A. J.; Bingham, M. Angew. Chem, Int. Ed., 2006, 45, 2664. (h) T. J. Donohoe, L. P. Fishlock and P. A. Procopiou, Chem.- Eur. J., 2008, 14, 5716.

8 (a) K. Yoshida, F. Kawagoe, K. Hayashi, S. Horiuchi, T. Imamoto and A. Yanagisawa, Org. Lett., 2009, 11, 515. (b) O. Fujimura, G. C. Fu and R. H. Grubbs, J. Org. Chem., 1994, 59, 4029.

9 T. J. Donohoe, A. Ironmonger and N. M. Kershaw, Angew. Chem. Int. Ed., 2008, 47, 7314.

10 A. K. Chatterjee, T.-L. Choi, D. P. Sanders and R. H. Grubbs, J. Am. Chem. Soc., 2003, 125, 11360.

11 (a) T. J. Donohoe, J. F. Bower, Proc. Natl. Acad. Sci. U.S.A., 2010, 107, 3373. (b) T. J. Donohoe, J. F. Bower and J. A. Basutto, Nature: Protocols, 2010, 5, 2005.

12 A. H. Hoveyda, P. J. Lombardi, R. V. O'Brien and A. R. Zhugralin, J. Am. Chem. Soc., 2009, 131, 8378.

13 Geometric inversion of the alkene during the Heck reaction has previously been reported: A. F. Littke and G. C. Fu, J. Am. Chem. Soc., 2001, 123, 6989.

14 T. J. Donohoe, N. J. Race, J. F. Bower and C. K. A. Callens, Org. Lett., 2010, 12, 4094.

15 S. Shafi, M. Kędziorek and K. Grela, Synlett, 2011, 124.

16 (a) T. J. Donohoe, J. A. Basutto, J. F. Bower and A. Rathi, Org. Lett., 2011, 13, 1036. (b) T. J. Donohoe, J. F. Bower, D. B. Baker, J. A.
Basutto, L. K. M. Chan and P. Gallagher, Chem. Commun., 2011, 47, 10611.

17 (a) L. Alcaraz, J. J. Hamett, C. Mioskowski, J. P. Madel, T. Le Gall, D.-S. Shin and J. R. Falck, Tetrahedron Lett., 1994, 35, 5449. (b) A. Fürstner, M. D. B. Fenster, B. Fasching, C. Godbout and K. Radkowski, Angew. Chem., 2006, 118, 5636; Angew. Chem. Int. Ed., 2006, 45, 5510. (c) H. Benakki, E. Colacino, C. Andre, F. Guenoun, J. Martinez, and F. Lamaty, Tetrahedron, 2008, 64, 5949. (d) M. Arisawa, C. Theeraladanon, A. Nishida, M. Nakagawa, Tetrahedron Lett., 2001, 42, 8029.

18 B. D. Roth, US Patent 5,273,995.

19 (a) A. Fürstner and A. Leitner, Angew. Chem. Int. Ed., 2003, 42, 308. (b) H. Hagiwara, T. Katsumi, V. P. Kamat, T. Hoshi, T. Suzuki and M. Ando, J. Org. Chem., 2000, 65, 7231.

20 (a) S.-Y. Wang, S.-J. Ji and T.-P. Loh, J. Am. Chem. Soc., 2007, 129, 276. (b) S.-Y. Wang, T.-K. Lum, S.-J. Ji and T.-P. Loh, Adv. Synth. Catal., 2008, 350, 673. 